\title{
Rigid rotor in phase space
}

\author{
S. Danko Bosanac \\ R. Boskovic Institute \\ 10001 Zagreb, Croatia
}

\begin{abstract}
Angular momentum is important concept in physics, and its phase space properties are important in various applications. In this work phase space analysis of the angular momentum is made from its classical definition, and by imposing uncertainty principle its quantum properties are obtained. It is shown that kinetic energy operator is derived, but it has different interpretation of its parts than in the standard treatment. Rigid rotor is discussed and it is shown what is its phase space representation. True rigid rotor is defined and also its phase space properties are discussed.
\end{abstract}

\section{INTRODUCTION}

Angular momentum is a very important concept in dynamics of particles and within quantum mechanics its properties are very well understood [1]. One example where the theory has direct application is the rigid rotor model, which is basic for understanding rotational spectroscopy and collisions of molecules. These processes are described by quantum dynamics, but there are circumstances when classical dynamics is used as alternative. For example, rotational cross sections for two colliding molecules in principle can be calculated from quantum mechanics, but often it is a challenging task. Classical mechanics,on the other hand, is relatively simple to use, but there several problems in its implementation, say to calculate atom-molecule collision cross sections. The basic one is how the initial conditions are selected and the final results analyzed. For example, if one says that a molecule is in the rotational state with the quantum numbers $l=3$ and $m=2$ then the question is what to choose for the initial orientation and angular velocity which adequately represents it? Analogous problem had been analyzed for collisions involving only vibrational energy exchange in atom-molecule collisions, where it was shown how to select initial conditions that adequately represent a particular vibrational state of the molecule. A recipe was suggested from which transition probabilities were successfully calculated [2] from classical mechanics, and by that it is meant that initial conditions were selected from a prescribed phase space density but dynamics is calculated from classical equations of motion.

The problem is therefore how does one chooses a set of initial positions and velocities for a particle so that on average its angular momentum has given $l$ and $m$ values? In other words, what is needed is a function $f_{l, m}(\vec{r}, \vec{p})$ with a property that the following averages are obtained 


$$
\begin{aligned}
& <(\vec{r} \times \vec{p})^{2}>=\int d^{3} r d^{3} p(\vec{r} \times \vec{p})^{2} f_{l, m}(\vec{r}, \vec{p})=\hbar^{2} l(l+1) \\
& <\vec{r} \times \vec{p}>=\int d^{3} r d^{3} p \vec{r} \times \vec{p} f_{l, m}(\vec{r}, \vec{p})=\hbar m \hat{z}
\end{aligned}
$$

In classical mechanics the function $f_{l, m}(\vec{r}, \vec{p})$ is interpreted as a statistical weight from which the position and momentum are selected. It should be noted that it is not necessary that $f_{l, m}(\vec{r}, \vec{p})$ is positive function, as long as those averages are obtained. In practice this means that initial conditions are randomly selected from $\left|f_{l, m}(\vec{r}, \vec{p})\right|$ and if $N$ sets are chosen then the averages are approximately

$$
<(\vec{r} \times \vec{p})^{2}>\approx \frac{\sum_{j=1}^{N}\left(\vec{r}_{j} \times \vec{p}_{j}\right)^{2} \operatorname{sign}\left[f_{l, m}\left(\vec{r}_{j}, \vec{p}_{j}\right)\right]}{\sum_{j=1}^{N} \operatorname{sign}\left[f_{l, m}\left(\vec{r}_{j}, \vec{p}_{j}\right)\right]}
$$

and similarly for the angular momentum. The function $\operatorname{sign}\left[f_{l, m}\left(\vec{r}_{j}, \vec{p}_{j}\right)\right]$ is the sign of $f_{l, m}\left(\vec{r}_{j}, \vec{p}_{j}\right)$ for the set of initial conditions $\left(\vec{r}_{j}, \vec{p}_{j}\right)$. However, much more stringent condition is that the function $f_{l, m}(\vec{r}, \vec{p})$ is stationary. The meaning of this can demonstrated on simple example. If the particle of mass $M$ is free and the set of its initial conditions is $\left(\vec{r}_{j}, \vec{p}_{j}\right)$ then its position after time $t$ is $\vec{r}=\vec{r}_{j}+\frac{\vec{p}_{j}}{M} t$ and velocity $\vec{v}=\frac{\vec{p}_{j}}{M}$. From these values one would be able to calculate a new distribution function $g(\vec{r}, \vec{p})$, by requiring to have properties as previously described for the function $f_{l, m}(\vec{r}, \vec{p})$. Stationarity requires that the two functions are identical for any time $t$. This restriction is very important, for obvious reasons, and it is sufficient to dismiss a large number of ad-hoc distributions.

The most obvious starting point would be the wave function for a particle in a particular angular momentum state, which in the coordinate space is $\psi_{l, m}(\vec{r})=\chi_{n, l}(r) Y_{l, m}(\theta, \phi)$, where $Y_{l, m}(\theta, \phi)$ is spherical harmonic. Likewise in momentum space the wave function is $\varphi_{l, m}(\vec{p})=$ $\omega_{n, l}(p) Y_{l, m}\left(\theta_{p}, \phi_{p}\right)$, where $\theta_{p}$ and $\phi_{p}$ are angles of the momentum. One could then form the function $f_{l, m}(\vec{r}, \vec{p})=\left|\chi_{n, l}(r) Y_{l, m}(\theta, \phi)\right|^{2}\left|\omega_{n, l}(p) Y_{l, m}\left(\theta_{p}, \phi_{p}\right)\right|^{2}$ from which initial position vector and momentum could be selected, except that neither the averages (11) are obtained nor the condition of stationarity for the function $f_{l, m}(\vec{r}, \vec{p})$ is satisfied, which is easily proven. Therefore this simple idea fails, and this is because one basic difference between classical and quantum dynamics was not taken into account: classical dynamics is defined in the phase space, while quantum dynamics is either in the coordinate or momentum space. Simple product that was suggested for the distribution $f_{l, m}(\vec{r}, \vec{p})$ only reflects this: it says that initial conditions in the phase space are determined as the product of the distribution from the coordinate and momentum space, as being independent. What is needed is formulation of quantum mechanics in the phase space, and then one would possibly be able to satisfy those two conditions. The earliest attempt in this direction was done by Wigner [3] who looked for a function $\rho(\vec{r}, \vec{p}, t)$ with the property

$$
P(\vec{r}, t)=|\psi(\vec{r}, t)|^{2}=\int d^{3} p \rho(\vec{r}, \vec{p}, t) \quad ; \quad Q(\vec{p}, t)=|\varphi(\vec{p}, t)|^{2}=\int d^{3} r \rho(\vec{r}, \vec{p}, t)
$$

and obtained, what is known as, the Wigner function

$$
\rho(\vec{r}, \vec{p}, t)=\frac{1}{\pi^{3} \hbar^{3}} \int d^{3} q e^{2 i \vec{p} \cdot \vec{q} / \hbar} \psi^{*}(\vec{r}+\vec{q}, t) \psi(\vec{r}-\vec{q}, t)
$$

which is regarded as extension of quantum mechanics into the phase space. By defining it in this way inevitably results in non uniqueness of the extension [4], because there are 
a large class of phase space functions that are defined by the requirements (2). Another attempt to formulate quantum mechanics in the phase space is due to Moyal [5,6] whose main objective was to give it a sound statistical foundation. The main starting point is postulating observables as operators, and postulating that to each set of commutating observables there is a set of non commuting. Together they form a complete set of observables. For these observable (operators) one forms a function from which the characteristic function of statistical theory for a given quantum state $\psi$ is obtained as a matrix element. By the Fourier transform Moyal obtains distribution which specifies to the Wigner function if the observables are coordinates and momenta. Common to both approaches is that the principles of quantum mechanics are assumed, although Moyal analysis is more general. Can the Wigner function be used for the distribution $f_{l, m}(\vec{r}, \vec{p})$ ? It is not clear that the averages (11) are obtained, in fact as it will be shown later they are only partly obtained. Furthermore, it is also not clear that the stationarity condition is satisfied, however, the Wigner function is stationary under the quantum time evolution for the stationary wave function, which is $\psi(\vec{r}, t)=\psi_{0}(\vec{r}) e^{-i E t / \hbar}$. Wigner function was used in various applications and its properties investigated [7 21], but its true significance is when connection with classical dynamics is sought. It is achieved by the standard assumption that classical dynamics is the limit of quantum when $\hbar \rightarrow 0$. It can be shown in general (one dimensional problem is discussed for the moment) that the Wigner function satisfies the equation [19,20

$$
\frac{\partial \rho}{\partial t}+\frac{p}{m} \frac{\partial \rho}{\partial x}+\frac{\partial V}{\partial x} \frac{\partial \rho}{\partial p}=-\frac{1}{2 i} \int_{-\infty}^{\infty} d y e^{i p y} G(x, y) \psi^{*}(x-\hbar y / 2, t) \psi(x+\hbar y / 2, t)
$$

where

$$
G(x, y)=\sum_{n=1}^{\infty} \frac{V^{(2 n+1)}(x)}{2^{2 n}(2 n+1) !}(\hbar y)^{2 n+1}
$$

and in this limit the inhomogeneous term vanishes. It also vanishes if the potential is quadratic, irrespective of this limit. The homogeneous equation is the Liouville equation, which determines time evolution of the phase space density $\rho(\vec{r}, \vec{p}, t)$, and it is solved by classical equations of motion.

Despite this, what appears, very important connection between quantum and classical dynamics, there are at least two remarks that one can make about this approach. It is desirable from understanding quantum-classical connection to learn more about foundations of quantum principles because with classical we are quite familiar. Strictly speaking that goal was not achieved because wether the Wigner or Moyal formulation of the phase space nothing can be learned about quantum principles because they are the starting point anyway. The second remark concerns the limit of the phase space density when $\hbar \rightarrow 0$, which should also be taken and not only the limit for the inhomogeneous term. For the stationary states it can be shown that 13

$$
\rho(\vec{r}, \vec{p}) \underset{\hbar \rightarrow 0}{=} \delta\left(H-E_{0}\right)=\delta\left[\frac{p^{2}}{2 m}+V(r)-E_{0}\right]
$$

where $E_{0}$ is a fixed energy. This form greatly restricts possible phase space densities from among those stationary solutions that are obtained from the Liouville equation. However, 
it is consistent with the accepted view which is based on the correspondence principle: quantum goes over to classical solution for large quantum numbers (the proof of this is straightforward but not elaborated).

Classical-quantum connection can be solved by starting from entirely classical principles [22,23], and as it will be shown allows much greater flexibility in application of classical dynamics to quantum problems. One starts from the Liouville equation in classical dynamics, and the argument on which one basis its use is quite straightforward: initial conditions for a particle are never accurately determined, in which case its precise trajectory has no meaning, only probabilities where it is found in the course of time. For example, to claim that position of Earth is accurately determined is nonsense, and therefore prediction of its position accurately in the next, say, million years is not possible, especially if perturbation from other planets is taken into account. However, it is possible to predict probability of finding it at certain position after (almost) any length of time. Therefore one starts by formulating classical mechanics as a statistical theory (it should be strongly emphasized that statistical does not imply many particles but probability for a single particle), in contrast to the attempt by Moyal whose principal aim was to formulate quantum mechanics as a statistical theory. In other words, one particle in the phase space is not a point but an extended density, which takes into account all uncertainties in determining its whereabouts. For a particle of mass $m$ this equation is

$$
\partial_{t} \rho(\vec{r}, \vec{p}, t)+\frac{\vec{p}}{m} \cdot \nabla_{r} \rho(\vec{r}, \vec{p}, t)+\vec{F} \cdot \nabla_{p} \rho(\vec{r}, \vec{p}, t)=0
$$

where $\vec{F}$ is the force on the particle and $\vec{p}$ is its momentum. The index of the operator nabla designates the variable with respect to which the derivatives are taken. For a particular case when phase space density is stationary, which means that $\partial_{t} \rho(\vec{r}, \vec{p}, t)=0$, the solution is a function of the form $\rho(\vec{r}, \vec{p}, t)=f\left(h_{1}, h_{2}, h_{3}, \ldots\right)$, where $f$ is arbitrary function and $h_{i}$ are dynamic invariants of the classical equations of motion. One of them is Hamiltonian for the particle, and another are components of the angular momentum if the force $\vec{F}$ is centrally symmetric. There are other invariants but they will be mentioned later. These phase space densities, like any other solution of the Liouville equation, lack a very important ingredient in order to be regarded also quantum solutions. This ingredient is restriction on the possible phase space densities, and it is in the form of the uncertainty principle

$$
\Delta x \Delta p_{x} \succeq \hbar / 2
$$

for any Cartesian coordinate. The standard deviations $\Delta x$ and $\Delta p$ are calculated from the phase space density. Mathematically speaking, selecting phase space densities according to this restriction is a very well defined problem and it is solved within the Fourier analysis. They are obtained in the form of the convolution

$$
\rho(\vec{r}, \vec{p}, t)=\frac{1}{\pi^{3} \hbar^{3}} \int d^{3} q e^{2 i \vec{p} \cdot \vec{q} / \hbar} f^{*}(\vec{r}+\vec{q}, t) f(\vec{r}-\vec{q}, t)
$$

which is also recognized as the Wigner function. The function $f$ is arbitrary, but if the phase space density satisfies the Liouville equation then it satisfies the equation [24]

$$
i \hbar \partial_{t} f=-\frac{\hbar^{2}}{2 m} \Delta f+V f
$$


where $\vec{F}=-\nabla V$, and for potential it is not required to be harmonic (for a general potential parametrization of the phase space density is more elaborate).

The common point between the Wigner and Moyal approach and the one that starts from classical principles is the function (3) or (5), however it is almost arbitrary (because there is no reason to choose it from many other functions) function in the former case but it is convolution in the latter. Apart from that common point there are fundamental differences between the two approaches, the former starts from quantum principles and the latter from classical. One manifestation of that is attitude towards the uncertainty relationship, which in the former case it is not considered a principle because it is derived from the other ones, while in the latter case it is assumed to be a fundamental principle from which the basic dynamics equation of quantum mechanics (6) is derived. One could cite other fundamental differences between the two approaches, but one is that of simplicity. In the Wigner-Moyal approach one needs all postulates as in formulation of quantum mechanics, about whose number there is no general consensus, but in the approach from classical mechanics one needs only the postulates that defines it (i.e. there is no need for postulates other than the ones that one is already familiar width) and the uncertainty postulate. One could question the meaning of the uncertainty principle, but one could equally question the meaning of the observable-operator, or wave-particle dualism postulates in the formulation of quantum mechanics. Other advantages that one has by starting from the classical principles and not from the quantum phase space formulation of Wigner and Moyal, will be explicitly manifested in the following sections. In particular it is not clear how to derive the phase space density for the true rigid rotor, which is done in this work, by other than starting from the classical principles.

There is one conceptual problem in the attempt to merge classical dynamics with the uncertainty principle. Without the latter solutions of the Liouville equation are the phase space probability densities, and as such they always have positive value. As soon as one imposes restriction in the form of the uncertainty principle this feature is lost and one often ends with the phase space density that has positive and negative values. This is the price it is paid by imposing that restriction, and its physics is justified on the grounds that the uncertainty principle makes it impossible to measure precisely the phase space probability density. One talks then about the phase space density, whose properties are exactly the same as for the probability density. However, all measurable quantities, e.g. probability density for the coordinates only, must have physically acceptable value.

In the equation (6) one recognizes Schroedinger equation, the basic equation of quantum mechanics. Therefore the described steps in classical mechanics produce identical results as quantum mechanics, provided the initial condition for the phase space density is calculated from the parameterization (5). From these principles angular momentum for the three dimensional harmonic oscillator will be analyzed first.

\section{HARMONIC OSCILLATOR IN THREE DIMENSIONS}

Stationary phase space densities for a three dimensional harmonic oscillator will be analyzed if it is assumed that the average

$$
\vec{L}=\int d^{3} r d^{3} p \vec{r} \times \vec{p} \rho(\vec{r}, \vec{p})
$$


has a given value. The starting point is the phase space density which is parametrized as (5), where the functions $\psi$ are chosen in the form $\psi=r^{l} Y_{l, \mu}(\theta, \phi) R_{n, l}(r)=Y_{l, \mu}(x, y, z) R_{n, l}(r)$, where $\theta$ and $\phi$ are spherical angles and $r$ is the radial coordinate. The indices $n, l$ and $\mu$ are integers (in further analysis the units are set in which $m=\hbar=1$ and the frequency of the oscillator is $\omega=1), Y_{l, \mu}(\theta, \phi)$ is spherical harmonic and

$$
R_{n, l}(r)=N_{1} F_{1}\left(-n, l+\frac{3}{2} ; r^{2}\right) e^{-\frac{1}{2} r^{2}}
$$

where $N$ is normalization constant and ${ }_{1} F_{1}(a, b ; z)$ is hypergeometric function. The phase space density is now

$$
\begin{gathered}
\rho_{n, l, \mu}(\vec{r}, \vec{p})= \\
\frac{1}{\pi^{3}} \int d^{3} q e^{2 i \vec{p} \cdot \vec{q}} Y_{l, \mu}^{*}\left(x+q_{x}, y+q_{y}, z+q_{z}\right) R_{n, l}(|\vec{r}+\vec{q}|) \\
Y_{l, \mu}\left(x-q_{x}, y-q_{y}, z-q_{z}\right) R_{n, l}(|\vec{r}-\vec{q}|)
\end{gathered}
$$

which in general does not have simple explicit form, but it has few nice features. It is stationary, which means that its form does not changes with respect to the classical time evolution. In other words, if classical solution for the trajectory is

$$
\vec{r}=\vec{r}_{0} \cos (t)+\vec{p}_{0} \sin (t)
$$

then

$$
\rho(\vec{r}, \vec{p}, t)=\rho_{n, l, \mu}[\vec{r} \cos (t)-\vec{p} \sin (t), \vec{r} \sin (t)+\vec{p} \cos (t)]=\rho_{n, l, \mu}(\vec{r}, \vec{p})
$$

This means that the phase space density is a function of the dynamic invariants of the harmonic oscillator. One set of these are elements of the energy tensor $\left(\frac{1}{2}\right.$ is omitted for simplicity) $E_{i, j}=p_{i} p_{j}+x_{i} x_{j} ; i, j=1,2,3$ (the indices designate the Cartesian components $x, y$ and $z$ ), and the other are the components of the angular momentum $x_{i} p_{j}-x_{j} p_{i} ; i \neq j$. Of course, any other combination of these basic invariants is possible, e.g. the total angular momentum squared. Which ones are present in the phase space density (8) are determined by explicit calculation, and the first few for the ground vibrational $(\mathrm{n}=0)$ state are given in Table I. The symbols represent the following quantities: $E=p^{2}+r^{2}, E_{z}=p_{z}^{2}+z^{2}, L^{2}=$ $\left(y p_{z}-z p_{y}\right)^{2}+\left(z p_{x}-x p_{z}\right)^{2}+\left(x p_{y}-y p_{x}\right)^{2}$ and $L_{z}=x p_{y}-y p_{x}$. These are dynamic invariants for the harmonic oscillator, and therefore the phase space density is indeed stationary.

From the phase space densities one can calculate the total angular momentum (7) and its squared modulus from

$$
L^{2}=\int d^{3} r d^{3} p(\vec{r} \times \vec{p})^{2} \rho(\vec{r}, \vec{p})
$$

Their values are given in Table I and as expected angular momentum is the same as from quantum analysis: it has only the $\mathrm{z}$ component and its value is $\mu$. However, the angular momentum squared is not equal to $l(l+1)$, as expected from the quantum treatment, but differs by $3 / 2$. The same is true for other than $n=0$ states, as shown in Table II. In fact the most surprising finding is that the states with $l=0$, which are normally associated with the zero angular momentum, have the value $3 / 2$ for the angular momentum squared. The question is where this discrepancy comes from? The simplest answer is that classical analysis 
is not correct, because it surely must violate certain rules that are not consistent with the quantum mechanical ones. However, this answer is not correct, because if one writes the momentum squared as $p^{2}=p_{r}^{2}+p_{\theta}^{2}+p_{\phi}^{2}$, where the components of the momentum are given with respect to the vector $\vec{r}$, then its average for the function $\psi=\Theta(\theta, \phi) R(r)$ is

$$
\begin{aligned}
& <p^{2}>=\frac{1}{\pi^{3}} \int d^{3} r d p_{r} d p_{\theta} d p_{\phi}\left(p_{r}^{2}+p_{\theta}^{2}+p_{\phi}^{2}\right) \\
& \quad \int d q_{r} d q_{\theta} d q_{\phi} e^{2 i\left(p_{r} q_{r}+p_{\theta} q_{\theta}+p_{\phi} q_{\phi}\right)} \Theta^{*}\left(\theta_{+}, \phi_{+}\right) R\left(r_{+}\right) \Theta\left(\theta_{-}, \phi_{-}\right) R\left(r_{-}\right)
\end{aligned}
$$

where

$$
\begin{aligned}
r_{ \pm} & =\sqrt{\left(r \pm q_{r}\right)^{2}+q_{\theta}^{2}+q_{\phi}^{2}} ; \cos \theta_{ \pm}=\frac{\hat{z} \cdot(\vec{r} \pm \vec{q})}{|\vec{r} \pm \vec{q}|}=\frac{\left(r \pm q_{r}\right) \cos \theta \pm q_{\theta} \sin \theta}{\sqrt{\left(r \pm q_{r}\right)^{2}+q_{\theta}^{2}+q_{\phi}^{2}}} \\
e^{i \phi_{ \pm}} & =\frac{(\vec{r} \pm \vec{q}) \cdot(\hat{x}+i \hat{y})}{|\vec{r} \pm \vec{q}| \sin \theta_{ \pm}}=\frac{\left(r \pm q_{r}\right) \sin \theta \mp q_{\theta} \cos \theta \pm i q_{\phi}}{|\vec{r} \pm \vec{q}| \sin \theta_{ \pm}} e^{i \phi}
\end{aligned}
$$

After a straightforward, but lengthy, calculation of the integrals one obtains

$$
<p^{2}>=-\int d^{3} r \psi^{*}(\vec{r}) \frac{1}{r^{2}}\left[\frac{d}{d r}\left(r^{2} \frac{d}{d r}\right)+\frac{1}{\sin (\theta)} \frac{\partial}{\partial \theta}\left(\sin (\theta) \frac{\partial}{\partial \theta}\right)+\frac{1}{\sin ^{2}(\theta)} \frac{\partial^{2}}{\partial \phi^{2}}\right] \psi(\vec{r})
$$

which is the correct answer for the kinetic energy operator (up to a pre-factor, which was not taken into account). In the standard interpretation the angular part is then associated with the angular momentum squared operator, which indeed gives zero for angular momentum squared for the $l=0$ states. Therefore the procedure of deriving the kinetic energy operator is correct, but then the question is where the analysis that produced results in Tables I and II is inconsistent with the quantum interpretation? To answer this question one explicitly calculates the average of $p_{\Omega}^{2}=p_{\theta}^{2}+p_{\phi}^{2}$, which is the classical angular part of the momentum squared. For simplicity its average will be calculated for $l=0$ state.

By definition

$$
\begin{aligned}
& <p_{\Omega}^{2}>=\frac{1}{4 \pi^{4}} \int d^{3} r d p_{r} d p_{\theta} d p_{\phi}\left(p_{\theta}^{2}+p_{\phi}^{2}\right) \\
& \quad \int d q_{r} d q_{\theta} d q_{\phi} e^{2 i\left(p_{r} q_{r}+p_{\theta} q_{\theta}+p_{\phi} q_{\phi}\right)} R\left(\sqrt{\left(r+q_{r}\right)^{2}+q_{\theta}^{2}+q_{\phi}^{2}}\right) R\left(\sqrt{\left(r-q_{r}\right)^{2}+q_{\theta}^{2}+q_{\phi}^{2}}\right)
\end{aligned}
$$

where integration in $p_{r}$ and $q_{r}$ is calculated first, then $p_{\theta}^{2}$ and $p_{\phi}^{2}$ are replaced by derivatives in the variables $q_{\theta}$ and $q_{\phi}$ that act on the exponential function, respectively, and after partial integration in the same variables one obtains

$$
<p_{\Omega}^{2}>=-\frac{1}{16 \pi^{3}} \int d^{3} r d p_{\theta} d p_{\phi} \int d q_{\theta} d q_{\phi} e^{2 i\left(p_{\theta} q_{\theta}+p_{\phi} q_{\phi}\right)}\left(\partial_{q_{\theta}}^{2}+\partial_{q_{\phi}}^{2}\right)\left[R^{2}\left(\sqrt{r^{2}+q_{\theta}^{2}+q_{\phi}^{2}}\right)\right]
$$

After integration in the variables $p_{\theta}$ and $p_{\phi}$ the only non zero contribution is

$$
<p_{\Omega}^{2}>=-\frac{1}{4 \pi} \int d^{3} r R(r) \frac{1}{r} \partial_{r} R(r)
$$

and it is not zero. In fact the average of the angular momentum squared (which is $L^{2}=r^{2} p_{\Omega}^{2}$ ) is 


$$
<L^{2}>=-\int_{0}^{\infty} d r r^{3} R(r) \partial_{r} R(r)=\frac{3}{2}
$$

which is precisely the value in Tables I and II. Furthermore the result is independent of the radial function, which indicates that this is a universal number. In order to check the correctness of the result one calculates the average of the radial part of the momentum squared, and the result is

$$
<p_{r}^{2}>=-\frac{1}{4 \pi} \int d^{3} r R(r)\left[\partial_{r}^{2} R(r)+\frac{1}{r} \partial_{r} R(r)\right]
$$

Together with the angular part one obtains the radial part of the kinetic energy operator

$$
T_{r}=-\frac{1}{r^{2}} \partial_{r}\left(r^{2} \partial_{r}\right)
$$

which is the correct answer. Therefore, there is no discrepancy between quantum expression for the kinetic energy and classical treatment in this work, but the difference is in the interpretation of its fragments. According to results in this work one should interpret the radial kinetic energy operator as

$$
T_{r}=-\partial_{r}^{2}-\frac{1}{r} \partial_{r}
$$

while

$$
T_{\Omega}=-\frac{1}{r} \partial_{r}-\frac{1}{r^{2}}\left[\frac{1}{\sin (\theta)} \frac{\partial}{\partial \theta}\left(\sin (\theta) \frac{\partial}{\partial \theta}\right)+\frac{1}{\sin ^{2}(\theta)} \frac{\partial^{2}}{\partial \phi^{2}}\right]
$$

is operator for the angular momentum squared.

Further confirmation of this comes from calculating the average angular momentum explicitly. For $l=0$ states it is given by

$<\vec{L}>=<L_{z}>\hat{z}=\frac{\hat{z}}{4 \pi^{4}} \int d^{3} r d p_{r} d p_{\theta} d p_{\phi} r p_{\phi} \sin (\theta) \int d q_{r} d q_{\theta} d q_{\phi} e^{2 i\left(p_{r} q_{r}+p_{\theta} q_{\theta}+p_{\phi} q_{\phi}\right)} R\left(r_{+}\right) R\left(r_{-}\right)$

which is easily shown to be zero. This is no contradiction with the previous finding, because zero of the angular momentum is result of cancellations of the contributions from two signs of $p_{\phi}$ rather than the modulus of the angular momentum being zero. One can therefore calculate angular momentum by averaging over only one sign of $p_{\phi}$, because the average over the other gives the same result but with the opposite sign, and the sum-total is zero. This average is

$$
<L_{z}^{+}>=\frac{1}{4 \pi^{4}} \int d^{3} r d p_{r} d p_{\theta} \sin (\theta) \int_{0}^{\infty} d p_{\phi} r p_{\phi} \int d q_{r} d q_{\theta} d q_{\phi} e^{2 i\left(p_{r} q_{r}+p_{\theta} q_{\theta}+p_{\phi} q_{\phi}\right)} R\left(r_{+}\right) R\left(r_{-}\right)
$$

and after evaluating most of integrals one gets

$$
<L_{z}^{+}>=-\frac{1}{8 i \pi^{2}} \int d^{3} r r \sin (\theta) \int_{0}^{\infty} d p_{\phi} \int d q_{\phi} e^{2 i p_{\phi} q_{\phi}} \partial_{q_{\phi}} R^{2}\left(\sqrt{r^{2}+q_{\phi}^{2}}\right)
$$

By using the relationship 


$$
\int_{0}^{\infty} d p_{\phi} e^{2 i p_{\phi} q_{\phi}}=\pi \delta\left(2 q_{\phi}\right)+i \boldsymbol{\Upsilon}\left(\frac{1}{2 q_{\phi}}\right)
$$

where 9 designates the principal value of the integral, it is obtained

$$
<L_{z}^{+}>=-\frac{i}{8 i \pi^{2}} \int d^{3} r r \sin (\theta) \boldsymbol{\Phi}\left[\int d q_{\phi} R\left(\sqrt{r^{2}+q_{\phi}^{2}}\right) R^{\prime}\left(\sqrt{r^{2}+q_{\phi}^{2}}\right) \frac{1}{\sqrt{r^{2}+q_{\phi}^{2}}}\right]
$$

The principal value can be omitted because the integrand is not singular, in which case

$$
<L_{z}^{+}>=\frac{1}{4} \int d r r^{2} R^{2}(r)=\frac{1}{4}
$$

The value of the $\mathrm{z}$ component of the angular momentum, which gets contribution from the space $p_{\phi}>0$, is independent of the radial function $R(r)$, the same result as before.

\section{RIGID ROTOR}

Rigid rotor in classical physics is a very well defined object, but one could argue that it is also in quantum. Unfortunately, as it will be shown, the two definitions have not the same meaning. Intuition, which is essentially classical, defines rigid object as the one which does not have breathing (radial) motion. Objects that appear to qualify as the rigid are diatomic molecules (at least many of them), which is supported by the approximations in their quantum description. Schroedinger equation for the nuclear motion in the diatomic molecule is

$$
i \hbar \frac{\partial \psi}{\partial t}=-\frac{\hbar^{2}}{2 m}\left[\frac{1}{r^{2}} \frac{\partial}{\partial r}\left(r^{2} \frac{\partial \psi}{\partial r}\right)+\frac{1}{r^{2} \sin (\theta)} \frac{\partial}{\partial \theta}\left(\sin (\theta) \frac{\partial \psi}{\partial \theta}\right)+\frac{1}{r^{2} \sin ^{2}(\theta)} \frac{\partial^{2} \psi}{\partial \phi^{2}}\right]+V(r) \psi
$$

where $m$ is the reduced mass of the diatomic molecule and $V(r)$ is the internuclear potential. Typically this potential has a deep minimum displaced from the origin by the distance $r_{0}$ (the bond length), which for the modelling purpose is approximated by $\frac{m}{2} \omega^{2}\left(r-r_{0}\right)^{2}$. Ground state wave function in this potential has the width $a$, and its relationship to the bond length is $r_{0} \gg a$. Under this circumstance the radial coordinate in the angular part of the kinetic energy operator is approximated by the fixed value $r_{0}$, in which case the wave function factors as $\psi=\frac{1}{r} R(r) \Theta(\theta, \phi, t) e^{-i E_{0} t / \hbar}$, where $E_{0}$ is the ground state energy of the molecule. The angular function then satisfies the equation

$$
i \hbar \frac{\partial \Theta(\theta, \phi, t)}{\partial t}=T_{r o t} \Theta(\theta, \phi, t)+W(\theta, \phi, t) \Theta(\theta, \phi, t)
$$

where

$$
T_{\text {rot }}=-\frac{\hbar^{2}}{2 m r_{0}^{2}}\left[\frac{1}{\sin (\theta)} \frac{\partial}{\partial \theta}\left(\sin (\theta) \frac{\partial}{\partial \theta}\right)+\frac{1}{\sin ^{2}(\theta)} \frac{\partial^{2}}{\partial \phi^{2}}\right]
$$

and $W(\theta, \phi, t)$ represents interaction of the molecule that depends on the angles only (say a dipole in the electric field). 
The unique feature of the equation (10) is that it depends only on the angle variables, orientations angles of the molecule, and in this sense it is called the equation for the rigid rotor. The operator $T_{\text {rot }}$ then represents the kinetic energy operator for the rigid rotor, which has discrete spectrum

$$
T_{\text {rot }} Y_{l, m}(\theta, \phi)=\frac{\hbar^{2}}{2 m r_{0}^{2}} l(l+1) Y_{l, m}(\theta, \phi) \quad ; \quad l=0,1,2, . . \quad m=-l,-l+1, \ldots, l
$$

where $Y_{l, m}(\theta, \phi)$ are spherical harmonics, the eigenfunctions of the rigid rotor. However, this is far from being true, if one has the image of the classical rigid rotor in mind. Although in the previous derivation dynamics in the angle variables is de-coupled from the dynamics in the radial this is not sufficient to call the molecule a rigid rotor. It is also necessary to prove that the radial component of the momentum for the relative motion of the two atoms is de-coupled from its angular ones, and to show this one needs to calculate the momentum space wave function. This function for a stationary rotational state of the "rigid rotor" is

$$
\varphi(\vec{p})=\int d^{3} r \frac{1}{r} R(r) Y_{l, m}(\theta, \phi) e^{i \vec{p} \cdot \vec{r}}=(2 \pi)^{3 / 2} i^{l} Y_{l, m}\left(\theta_{p}, \phi_{p}\right) \frac{1}{\sqrt{p}} \int_{0}^{\infty} d r r^{1 / 2} R(r) J_{l+1 / 2}(p r)
$$

where the expansion of the plane wave in the spherical coordinates was used. De-coupling of the radial component $p_{r}$ from its angular ones $p_{\theta}$ and $p_{\phi}$ is therefore not possible because $p=\sqrt{p_{r}^{2}+p_{\theta}^{2}+p_{\phi}^{2}}$, while the spherical angles $\theta_{p}$ and $\phi_{p}$ are related to the same components in a complicated way. Therefore it cannot be assumed that the theoretical model that was just described represents rigid rotor.

Analysis in the phase space is more revealing, but it is somewhat approximate. It was mentioned that around $r=r_{0}$ the potential is very well approximated by harmonic, in which case the entire wave function for the diatomic molecule is

$$
\phi_{l}(\vec{r})=N e^{-\frac{\left(r-r_{0}\right)^{2}}{2 a^{2}}} Y_{l, m}(\theta, \phi)
$$

where $1 / r$ coefficient was assumed to be constant. The phase space density is then

$$
\rho_{l, m}(\vec{r}, \vec{p})=\frac{N^{2}}{\pi^{3}} \int d^{3} q e^{2 i \vec{p} \cdot \vec{q}} Y_{l, m}^{*}\left(\theta_{+}, \phi_{+}\right) Y_{l, m}\left(\theta_{-}, \phi_{-}\right) e^{-\frac{\left(|\vec{r}+\vec{q}|-r_{0}\right)^{2}}{2 a^{2}}-\frac{\left(|\vec{r}-\vec{q}|-r_{0}\right)^{2}}{2 a^{2}}}
$$

where the angles $\theta_{ \pm}$and $\phi_{ \pm}$are defined in (9). The rigid rotor model assumption implies that $q<<r$, and if spherical coordinates are used for the vector $\vec{q}=q_{r} \hat{r}+q_{\theta} \hat{\theta}+q_{\phi} \vec{\phi}$ then the exponent of the harmonic oscillator function is approximately

$$
\frac{\left(|\vec{r}+\vec{q}|-r_{0}\right)^{2}}{2 a^{2}}+\frac{\left(|\vec{r}-\vec{q}|-r_{0}\right)^{2}}{2 a^{2}} \sim \frac{\left(r-r_{0}\right)^{2}}{a^{2}}+\frac{q_{r}^{2}}{a^{2}}\left(1-\frac{q_{\theta}^{2}+q_{\phi}^{2}}{r_{0}^{2}}\right)+\frac{\left(q_{\theta}^{2}+q_{\phi}^{2}\right)^{2}}{4 a^{2} r_{0}^{2}}+O\left(q^{6}\right)
$$

where in the expansion coefficients, except in the leading one, it was set $r=r_{0}$. By recalling that this expansion is in the exponent it follows that the range of $\left|q_{r}\right|$ within which it significantly contributes to the phase space density is of the order $a$, however, the range of $\sqrt{q_{\theta}^{2}+q_{\phi}^{2}}$ is of the order $\sqrt{a r_{0}}$. This means that $\left|q_{r}\right|<<\sqrt{q_{\theta}^{2}+q_{\phi}^{2}}$ in which case the phase space density is approximately 


$$
\begin{aligned}
\rho_{l, m}(\vec{r}, \vec{p})= & \frac{2 r_{0} a^{2} N^{2}}{\pi^{5 / 2}} e^{-\frac{\left(r-r_{0}\right)^{2}}{a^{2}}-p_{r}^{2} a^{2}} \\
& \int_{-\infty}^{\infty} d q_{\theta} \int_{-\infty}^{\infty} d q_{\phi} e^{2 i \sqrt{2 a r_{0}}\left(p_{\theta} q_{\theta}+p_{\phi} q_{\phi}\right)-\left(q_{\theta}^{2}+q_{\phi}^{2}\right)^{2}} \Theta_{l, m}\left(r_{0}, \theta, \phi, 0, \sqrt{2 a r_{0}} q_{\theta}, \sqrt{2 a r_{0}} q_{\phi}\right)
\end{aligned}
$$

where the angular function $\Theta_{l, m}$ is shorthand for the product of two functions $Y_{l, m}\left(\theta_{ \pm}, \phi_{ \pm}\right)$. Dependence of the angular function on the radial variable $q_{r}$ is also neglected, in which case the phase space density parametrizes as a product of the function for the radial variables and the function for the angular. The angular phase space density is now defined as

$\rho_{l, m}^{\Omega}\left(\theta, \phi, p_{\theta}, p_{\phi}\right)=\frac{2 a r_{0}}{\pi^{2}} \int d q_{\theta} d q_{\phi} e^{2 i \sqrt{2 a r_{0}}\left(p_{\theta} q_{\theta}+p_{\phi} q_{\phi}\right)-\left(q_{\theta}^{2}+q_{\phi}^{2}\right)^{2}} \Theta_{l, m}\left(r_{0}, \theta, \phi, 0, \sqrt{2 a r_{0}} q_{\theta}, \sqrt{2 a r_{0}} q_{\phi}\right)$

and appears to describe the rigid rotor because it is independent of the radial variables. However, this conclusion is false because the phase space density depends on the radial parameter $a$, and in this respect it depends on the radial dynamics. Because of this feature the rigid rotor will be called "soft".

Average of the angular momentum squared has now the value

$$
<L^{2}>=\int \rho_{l, m}^{\Omega}\left(\theta, \phi, p_{\theta}, p_{\phi}\right) r_{0}^{2}\left(p_{\theta}^{2}+p_{\phi}^{2}\right)=l(l+1)
$$

where the constant term $3 / 2$ that was obtained in the previous section is missing. This is expected because coupling with the radial motion was neglected. Another property of the angular phase space density is

$$
\int_{-\infty}^{\infty} d p_{\theta} \int_{-\infty}^{\infty} d p_{\phi} \rho_{l, m}^{\Omega}\left(\theta, \phi, p_{\theta}, p_{\phi}\right)=\Theta_{l, m}\left(r_{0}, \theta, \phi, 0,0,0\right)=\left|Y_{l, m}(\theta, \phi)\right|^{2}
$$

which shows that it correctly describes the essentials of the angular momentum. Its explicit form for $l=m=0$ is given by

$$
\rho_{0,0}^{\Omega}\left(\theta, \phi, p_{\theta}, p_{\phi}\right)=\frac{a r_{0}}{\pi^{2}} \int_{0}^{\infty} d q q J_{0}\left(2 \sqrt{2 a r_{0}} p q\right) e^{-q^{4}}
$$

which does not have explicit analytic expression, but its shape is simple, as shown in Figure 1. For the rotational state $l=1$ and $m=0$ the angular phase space density is

$$
\begin{aligned}
\rho_{1,0}^{\Omega}\left(\theta, \phi, p_{\theta}, p_{\phi}\right) & =\frac{6 a r_{0}}{4 \pi^{3}} \int_{-\infty}^{\infty} d q_{\theta} \int_{-\infty}^{\infty} d q_{\phi} e^{2 i \sqrt{2 a r_{0}}\left(p_{\theta} q_{\theta}+p_{\phi} q_{\phi}\right)-\left(q_{\theta}^{2}+q_{\phi}^{2}\right)^{2}} \frac{\cos ^{2} \theta-2 \epsilon q_{\theta}^{2} \sin ^{2} \theta}{1+2 \epsilon q_{\theta}^{2}+2 \epsilon q_{\phi}^{2}} \\
& \sim\left(\cos ^{2} \theta+\frac{1}{4 r_{0}^{2}} \partial_{p_{\theta}}^{2}+\frac{\cos ^{2} \theta}{4 r_{0}^{2}} \partial_{p_{\phi}}^{2}\right) \int_{0}^{\infty} d q q J_{0}\left(2 \sqrt{2 a r_{0}} p q\right) e^{-q^{4}}
\end{aligned}
$$

where $\epsilon=a / r_{0}$, and in the last step only the terms up to the order $\epsilon$ where retained. Similarly the phase space density for $l=1$ and $m=1$ is

$\rho_{1,1}^{\Omega}\left(\theta, \phi, p_{\theta}, p_{\phi}\right) \sim\left(\sin ^{2} \theta+\frac{\sin \theta}{r_{0}} \partial_{p_{\phi}}+\frac{1}{4 r_{0}^{2}} \partial_{p_{\theta}}^{2}+\frac{1}{4 r_{0}^{2}} \partial_{p_{\phi}}^{2}+\frac{\sin ^{2} \theta}{4 r_{0}^{2}} \partial_{p_{\phi}}^{2}\right) \int_{0}^{\infty} d q q J_{0}\left(2 \sqrt{2 a r_{0}} p q\right) e^{-q^{4}}$ 
These phase space densities, except $\rho_{0,0}^{\Omega}$, are time dependent, which means that they are not functions of only the dynamic invariants of the rigid rotor, e.g. $p^{2}=p_{\theta}^{2}+p_{\phi}^{2}$ and $p_{\phi} \sin \theta$. Thus for example the terms in $\rho_{1,0}^{\Omega}\left(\theta, \phi, p_{\theta}, p_{\phi}\right)$ that cannot be represented by invariants are

$\rho_{1,0}^{\Omega}\left(\theta, \phi, p_{\theta}, p_{\phi}\right)=\cos ^{2} \theta \int_{0}^{\infty} d q q J_{0}\left(2 \sqrt{2 a r_{0}} p q\right) e^{-q^{4}}-\frac{\cos ^{2} \theta}{p r_{0}^{2}} \sqrt{\frac{a r_{0}}{2}} \int_{0}^{\infty} d q q^{2} J_{1}\left(2 \sqrt{2 a r_{0}} p q\right) e^{-q^{4}}$

Explicit time dependence of the phase space density is obtained from the time dependence of the angle $\theta$

$$
\cos \theta=\cos \theta_{0} \cos \frac{t p_{0}}{m r_{0}}-\frac{p_{\theta}^{0}}{p_{0}} \sin \theta_{0} \sin \frac{t p_{0}}{m r_{0}}
$$

where $\theta_{0}, p_{\theta}^{0}$ and $p_{0}$ are initial values of these variables. In the phase space density $\cos \theta$ is replaced by

$$
\cos \theta_{t}=\cos \theta \cos \frac{t p}{m r_{0}}+\frac{p_{\theta}}{p} \sin \theta \sin \frac{t p}{m r_{0}}
$$

and the time dependence of the probability density (13) is calculated from

$$
\begin{gathered}
\int_{-\infty}^{\infty} d p_{\theta} \int_{-\infty}^{\infty} d p_{\phi} \rho_{1,0}^{\Omega}\left(\theta, \phi, p_{\theta}, p_{\phi}\right) \sim \int_{-\infty}^{\infty} d p_{\theta} \int_{-\infty}^{\infty} d p_{\phi} \cos ^{2} \theta \int_{0}^{\infty} d q q J_{0}\left(2 \sqrt{2 a r_{0}} p q\right) e^{-q^{4}} \\
=\frac{\pi}{32 a r_{0}}\left(1+\cos ^{2} \theta\right)-\frac{\pi m r_{0}}{8 \sqrt{2 a r_{0}}}\left(3 \cos ^{2} \theta-1\right) \partial_{t} \int_{0}^{\frac{t}{m r_{0} \sqrt{2 a r_{0}}}} d q \frac{q e^{-q^{4}}}{\sqrt{\frac{t^{2}}{2 a m^{2} r_{0}^{3}}-q^{2}}}
\end{gathered}
$$

The first part is time independent, while the second goes to zero after the time interval $t>m r_{0} \sqrt{2 a r_{0}}$, which is typically of the order $10^{-13}-10^{-14}$ sec for diatomic molecules. Therefore the probability density starts as (13) but its limiting value is constant, but not in the form of the squared modulus of the spherical harmonic.

\section{TRUE RIGID ROTOR}

Previous discussion revealed great difficulty in formulating the concept of the rigid rotor in quantum theory. This was manifested as inability to formulate the phase space density that involves only the parameters for the rotational degrees of freedom. It does not help to take the limit $a \longrightarrow 0$ in (12) because that would imply infinite dispersion of the variables $p_{\theta}$ and $p_{\phi}$, which only reflects the fact that the radial and the angular components of the momentum are interrelated. There is, however, a way of formulating the true rigid rotor, but should be done by following formulation of quantum mechanics as suggested in Introduction. One starts from the Liouville equation in the spherical coordinates, which for a free particle is

$$
\begin{aligned}
& \partial_{t} \rho-\frac{p_{\theta}}{m r} \partial_{\theta} \rho+\frac{p_{\phi}}{m r \sin \theta} \partial_{\phi} \rho+\frac{p_{r}}{m} \partial_{r} \rho- \\
& \frac{1}{m r}\left(\frac{p_{\phi}^{2} \cos \theta}{\sin \theta}+p_{r} p_{\theta}\right) \partial_{p_{\theta}} \rho-\frac{p_{\phi}}{m r}\left(-p_{\theta} \frac{\cos \theta}{\sin \theta}+p_{r}\right) \partial_{p_{\phi}} \rho+\left(\frac{p_{\theta}^{2}}{m r}+\frac{p_{\phi}^{2}}{m r}\right) \partial_{p_{r}} \rho=0
\end{aligned}
$$


and the rigid rotor assumption implies that the phase space density is $r$ and $p_{r}$ independent. This means that the Liouville equation for the rigid rotor is

$$
\partial_{t} \rho-\frac{p_{\theta}}{m r} \partial_{\theta} \rho+\frac{p_{\phi}}{m r \sin \theta} \partial_{\phi} \rho+\frac{p_{\phi}}{m r} \frac{\cos \theta}{\sin \theta}\left(p_{\theta} \partial_{p_{\phi}} \rho-p_{\phi} \partial_{p_{\theta}} \rho\right)=0
$$

where $r$ is constant. It can be easily verified that if the phase space density is a function of the form $\rho\left(\theta, \phi, p_{\theta}, p_{\phi}, t\right)=F\left(p_{\theta}^{2}+p_{\phi}^{2}, p_{\phi} \sin \theta\right)$, where $F$ is arbitrary function, then it is time independent. Additional requirement is that the phase space density should be in accordance with the uncertainty principle, which is achieved by straightforward generalization of the rule that was used before. The phase space density is therefore parametrized as

$$
\rho\left(\theta, \phi, p_{\theta}, p_{\phi}, t\right)=\frac{1}{\pi^{2}} \int d^{2} q e^{2 i\left(p_{\theta} q_{\theta}+p_{\phi} q_{\phi}\right)} \Theta^{*}\left(\theta_{+}, \phi_{+}\right) \Theta\left(\theta_{-}, \phi_{-}\right)
$$

where the angular functions will be determined for a particular case when the solutions of the Liouville equation (14) are stationary, i.e. $\partial_{t} \rho=0$. The relevant variables were defined in (9). Parametrization (15) is replaced in the Liouville equation (14), and by using transformations of the kind

$$
\begin{aligned}
p_{\theta} \rho\left(\theta, \phi, p_{\theta}, p_{\phi}\right) & =-\frac{1}{2 i} \int d^{2} q e^{2 i\left(p_{\theta} q_{\theta}+p_{\phi} q_{\phi}\right)} \partial_{q_{\theta}}\left[\Theta\left(\theta_{-}, \phi_{-}\right) \Theta^{*}\left(\theta_{+}, \phi_{+}\right)\right] \\
p_{\theta} \partial_{p_{\theta}} \rho\left(\theta, \phi, p_{\theta}, p_{\phi}\right) & =-\int d^{2} q e^{2 i\left(p_{\theta} q_{\theta}+p_{\phi} q_{\phi}\right)} \partial_{q_{\theta}}\left[q_{\theta} \Theta\left(\theta_{-}, \phi_{-}\right) \Theta^{*}\left(\theta_{+}, \phi_{+}\right)\right]
\end{aligned}
$$

one obtains, after lengthy simplifications, that the stationary solutions of the Liouville equation (14) satisfy

$$
\frac{i}{2 \pi^{2}} \int d^{2} q e^{2 i\left(p_{\theta} q_{\theta}+p_{\phi} q_{\phi}\right)} \frac{1}{r^{2}+q^{2}}\left(f_{1}+\frac{1}{r} f_{2}\right)=0
$$

where

$$
f_{1}=\Theta^{*}\left[\frac{1}{\sin \theta_{-}} \partial_{\theta_{-}}\left(\sin \theta_{-} \partial_{\theta_{-}} \Theta\right)+\frac{1}{\sin ^{2} \theta_{-}} \partial_{\phi_{-}}^{2} \Theta\right]-\Theta\left[\frac{1}{\sin \theta_{+}} \partial_{\theta_{+}}\left(\sin \theta_{+} \partial_{\theta_{+}} \Theta^{*}\right)+\frac{1}{\sin ^{2} \theta_{+}} \partial_{\phi+}^{2} \Theta^{*}\right]
$$

and

$$
\begin{aligned}
f_{2}= & -\frac{q^{2}}{2 r}\left(\cos \theta_{+}+\cos \theta_{-}\right)\left(\frac{\Theta^{*} \partial_{\theta_{-}} \Theta}{\sin \theta_{-}}-\frac{\Theta \partial_{\theta_{+}} \Theta^{*}}{\sin \theta_{+}}\right)-\frac{r}{2}\left(\cos \theta_{+}-\cos \theta_{-}\right)\left(\frac{\Theta^{*} \partial_{\theta_{-}} \Theta}{\sin \theta_{-}}+\frac{\Theta \partial_{\theta_{+}} \Theta^{*}}{\sin \theta_{+}}\right)+ \\
& q_{\phi} \sin \theta\left(\frac{\Theta^{*} \partial_{\phi_{-}} \Theta}{\sin ^{2} \theta_{-}}+\frac{\Theta \partial_{\phi_{+}} \Theta^{*}}{\sin ^{2} \theta_{+}}\right)
\end{aligned}
$$

where $q^{2}=q_{\theta}^{2}+q_{\phi}^{2}$. It is implied that $\Theta$ is a function of the variables $\theta_{-}$and $\phi_{-}$while $\Theta^{*}$ is a function of $\theta_{+}$and $\phi_{+}$.

If the function $f_{2}$ is neglected for the moment then the condition (10) implies that the angular function satisfies the differential equation

$$
\frac{1}{\sin \theta} \partial_{\theta}\left(\sin \theta \partial_{\theta} \Theta\right)+\frac{1}{\sin ^{2} \theta} \partial_{\phi}^{2} \Theta=\lambda \Theta
$$


where $\lambda$ is a real constant. In this equation one recognizes the equation for the spherical harmonics, where $\lambda=-l(l+1)$. However, the function $f_{2}$ cannot be neglected, and therefore the angular functions are only approximately the spherical harmonics. However, a very useful feature of the function $f_{2}$ is that in the limits $q_{\theta} \rightarrow 0$ and $q_{\phi} \rightarrow 0$ it is equal to zero, in which case the angular functions are exactly the spherical harmonics. This means that when the phase space density is integrated over the momentum variables the resulting probability density should be the squared modulus of the spherical harmonics, i.e.

$$
P(\theta, \phi)=\int d p_{\theta} \int d p_{\phi} \rho\left(\theta, \phi, p_{\theta}, p_{\phi}\right)=|\Theta(\theta, \phi)|^{2}=\left|Y_{l, m}(\theta, \phi)\right|^{2}
$$

The choice of the spherical harmonics for the angular functions is that the phase space density is approximate, which is manifested as being time dependent, i.e. it is not a function of only the dynamic invariants of the rigid rotor. This is the price that is paid by neglecting the function $f_{2}$ in the equation (16). Inclusion of this function results in the phase space density that is a function of only these invariants, and this fact is used as the procedure to find a proper phase space density. This is best demonstrated on one example. One particular case, however, has exact solution, and this is when the angular function is constant. In this case

$$
\rho_{0,0}\left(\theta, \phi, p_{\theta}, p_{\phi}\right)=\frac{1}{\pi^{2}} \int d^{2} q e^{2 i\left(p_{\theta} q_{\theta}+p_{\phi} q_{\phi}\right)} \frac{1}{4 \pi}=\frac{1}{4 \pi} \delta\left(p_{\theta}\right) \delta\left(p_{\phi}\right)
$$

The example that will be analyzed in more details is when the angular function is the spherical harmonic $Y_{1,0}(\theta, \phi)$. The phase space density (15) is

$$
\rho\left(\theta, \phi, p_{\theta}, p_{\phi}, t\right)=\frac{1}{\pi^{2}} \int d^{2} q e^{2 i\left(p_{\theta} q_{\theta}+p_{\phi} q_{\phi}\right)} Y_{1,0}^{*}\left(\theta_{+}, \phi_{+}\right) Y_{1,0}\left(\theta_{-}, \phi_{-}\right)
$$

and its explicit form is (the constant $r$ is fixed to unity)

$$
\begin{aligned}
\rho\left(\theta, \phi, p_{\theta}, p_{\phi}, t\right)= & \frac{3}{4 \pi^{3}} \int d^{2} q e^{2 i\left(p_{\theta} q_{\theta}+p_{\phi} q_{\phi}\right)} \frac{q_{\theta}^{2} \sin ^{2} \theta-\cos ^{2} \theta}{1+q^{2}}= \\
& \frac{3}{2 \pi^{2}}\left(1-\frac{p_{\phi}^{2} \sin ^{2} \theta}{p^{2}}\right) K_{0}(2 p)+\frac{3 \sin ^{2} \theta}{4 p \pi^{2}}\left(1-2 \frac{p_{\phi}^{2}}{p^{2}}\right) K_{1}(2 p)-\frac{3}{8 \pi} \delta\left(p_{\theta}\right) \delta\left(p_{\phi}\right) \sin ^{2} \theta
\end{aligned}
$$

where $K_{n}(x)$ is modified Bessel function of the second kind. This phase space density is not stationary, because the term

$$
\rho_{t}=\frac{3 \sin ^{2} \theta}{4 p \pi^{2}} K_{1}(2 p)
$$

is not a combination of the dynamic invariants. However, if $p_{\phi}$ is replaced by $p \cos \alpha$ then

$$
\int_{0}^{2 \pi} d \alpha\left(1-2 \frac{p_{\phi}^{2}}{p^{2}}\right)=0
$$

which means that these two terms in the phase space density can be omitted without in any way modifying the integral (17), the value of the total angular momentum squared

$$
<L^{2}>=\int d \Omega \int d^{2} p p^{2} \rho\left(\theta, \phi, p_{\theta}, p_{\phi}\right)
$$


and the angular momentum (its z-th component)

$$
<\vec{L}>=\hat{z} \int d \Omega \int d^{2} p p_{\phi} \sin \theta \rho\left(\theta, \phi, p_{\theta}, p_{\phi}\right)=<L_{z}>\hat{z}
$$

Therefore

$$
\rho_{1,0}\left(\theta, \phi, p_{\theta}, p_{\phi}\right)=\frac{3}{2 \pi^{2}}\left(1-\frac{p_{\phi}^{2} \sin ^{2} \theta}{p^{2}}\right) K_{0}(2 p)-\frac{3}{8 \pi} \delta\left(p_{\theta}\right) \delta\left(p_{\phi}\right) \sin ^{2} \theta
$$

is time independent and represents phase space density for the rigid rotor in the state with the angular momentum squared $\left\langle L^{2}\right\rangle=2$ and angular momentum $\left\langle L_{z}\right\rangle=0$, while the probability density (17) is $P(\theta, \phi)=\left|Y_{1,0}(\theta, \phi)\right|^{2}$. Similarly the phase space density for $l=1$ and $m=1$ is

$$
\rho_{1,1}\left(\theta, \phi, p_{\theta}, p_{\phi}\right)=\frac{3}{4 \pi^{2}}\left(1+\frac{p_{\phi}^{2} \sin ^{2} \theta}{p^{2}}\right) K_{0}(2 p)+\frac{3 p_{\phi} \sin \theta}{2 \pi^{2} p} K_{1}(2 p)+\frac{3}{16 \pi}\left(-2+\sin ^{2} \theta\right) \delta\left(p_{\theta}\right) \delta\left(p_{\phi}\right)
$$

with the property that $P(\theta, \phi)=\left|Y_{1,1}(\theta, \phi)\right|^{2},<L^{2}>=2$ and $<L_{z}>=1$.

The phase space densities for the angular momentum states $(2, m) ; m=0,1,2$ were calculated as additional example. They are associated with the angular momentum squared value $\left\langle L^{2}\right\rangle=6$ and the angular momentum $\left\langle L_{z}\right\rangle=0,1,2$, respectively. They are

$$
\begin{aligned}
\rho_{2,0}\left(\theta, \phi, p_{\theta}, p_{\phi}\right)= & \frac{15}{4 \pi^{2}}\left(-1+\frac{p_{\phi}^{4} \sin ^{4} \theta}{p^{4}}\right) K_{0}(2 p)+\frac{45 p}{8 \pi^{2}}\left(1-\frac{p_{\phi}^{2} \sin ^{2} \theta}{p^{2}}\right)^{2} K_{1}(2 p)+ \\
& \frac{5}{128 \pi}\left(8-24 \sin ^{2} \theta+27 \sin ^{4} \theta\right) \delta\left(p_{\theta}\right) \delta\left(p_{\phi}\right) \\
\rho_{2,1}\left(\theta, \phi, p_{\theta}, p_{\phi}\right)= & \frac{15}{4 \pi^{2}}\left(-1+2 p_{\phi} \sin \theta+\frac{p_{\phi}^{2} \sin ^{2} \theta}{p^{2}}-2 p_{\phi}^{3} \sin ^{3} \theta-\frac{2 p_{\phi}^{4} \sin ^{4} \theta}{3 p^{4}}\right) K_{0}(2 p)+ \\
& \frac{15 p}{4 \pi^{2}}\left(1-2 \frac{p_{\phi}^{3} \sin ^{3} \theta}{p^{4}}-\frac{p_{\phi}^{4} \sin ^{4} \theta}{p^{4}}\right) K_{1}(2 p)+\frac{15}{16 \pi}\left(\sin ^{2} \theta-\frac{3}{4} \sin ^{4} \theta\right) \delta\left(p_{\theta}\right) \delta\left(p_{\phi}\right) \\
\rho_{2,2}\left(\theta, \phi, p_{\theta}, p_{\phi}\right)= & \frac{15}{8 \pi^{2}}\left(-1+2 p_{\phi} \sin \theta-2 \frac{p_{\phi}^{2} \sin ^{2} \theta}{p^{2}}+2 \frac{p_{\phi}^{3} \sin ^{3} \theta}{p^{2}}+\frac{p_{\phi}^{4} \sin ^{4} \theta}{3 p^{4}}\right) K_{0}(2 p)+ \\
& \frac{15 p}{16 \pi^{2}}\left(1-4 \frac{p_{\phi} \sin \theta}{p^{2}}+6 \frac{p_{\phi}^{2} \sin ^{2} \theta}{p^{2}}+\frac{4 p_{\phi}^{3} \sin ^{3} \theta}{3 p^{4}}+\frac{p_{\phi}^{4} \sin ^{4} \theta}{p^{4}}\right) K_{1}(2 p)+ \\
& \frac{15}{32 \pi}\left(1-\sin ^{2} \theta+\frac{3}{8} \sin ^{4} \theta\right) \delta\left(p_{\theta}\right) \delta\left(p_{\phi}\right)
\end{aligned}
$$

In this way phase space for the true rigid rotor was defined. It is "true" because only the variables that are relevant for such object were introduced.

\section{DISCUSSION}

Analysis of angular momentum in the phase space was made, and in this context rigid rotor was discussed. Perhaps one of the most intriguing finding is interpretation for the partition of the kinetic energy operator, which is in considerable disagreement with the 
standard one. Part of what is considered to be radial kinetic energy is in fact contribution from the angular momentum operator, despite the fact that it only contains radial variable. This finding does not come as a surprise if one makes the following observation in the traditional classical mechanics. Given spherically symmetric probability distribution of coordinates $P(r)$ and momenta $Q(p)$ for a free particle (say it was obtained my measuring its position and momentum) the average kinetic energy is not zero despite the fact that the momentum is. Likewise, the average angular momentum is zero but the average modulus squared of it is not because

$$
<L^{2}>=\int d^{3} r \int d^{3} p r^{2}\left(p_{\theta}^{2}+p_{\phi}^{2}\right) P(r) Q(p) \neq 0
$$

Introducing the uncertainty principle does not changes this fact, except that instead of this average having arbitrary value it has a fixed and equal to $3 / 2$. Therefore a particle has always non zero modulus of angular momentum, and its minimal value is fixed and independent of the phase space density. It is like saying that particle always carries a minimal intrinsic angular momentum, but in the way it is described it never manifests itself. For all practical purpose this finding is immaterial, because standard interpretation is self sufficient, but it becomes evident when classical modelling is attempted.

From the phase space density for the rigid rotor one expects to obtain momentum space probability density, which is given by

$$
Q(\vec{p})=\int d^{3} r \rho(\vec{r}, \vec{p})
$$

however one should be careful about components of the momentum variable. Throughout the paper the components with respect to the vector $\vec{r}$ were used, because they are natural when angular momentum is analyzed. This is because $p_{\theta}$ and $p_{\phi}$ are components along the appropriate angular unit vectors that are perpendicular to the vector $\vec{r}$ and hence directly proportional to the angular momentum (they can be called radius vector components of the momentum). However, in the momentum space one works only with the components of the momentum vector, which for the mentioned components this is not the case. If $p$ is modulus of the vector $\vec{p}$ while $\theta_{p}$ and $\phi_{p}$ are its spherical angles then

$$
\begin{aligned}
& p_{r}=p\left(\cos \theta \cos \theta_{p}+\cos \left[\phi-\phi_{p}\right] \sin \theta \sin \theta_{p}\right) \\
& p_{\theta}=p\left(\sin \theta \cos \theta_{p}-\cos \left[\phi-\phi_{p}\right] \cos \theta \sin \theta_{p}\right) \\
& p_{\phi}=-p \sin \left[\phi-\phi_{p}\right] \sin \theta_{p}
\end{aligned}
$$

which explicitly shows that the radius vector components of momentum are a mixture of momentum and radial vector spherical coordinates. Therefore in the phase space density they must be replaced by (18) and then the integration over the spatial coordinates performed. Indeed for the "soft" rigid rotor it can be shown that one obtains for the momentum distribution the square modulus of (11), but it is not clear what the outcome would be for the true rigid rotor. For the latter it is required that $p_{r}=0$ and yet the expression for the phase space density would be a function of $p$ and $\theta_{p}$ without an obvious restriction of that kind. However, explicit expression for the momentum probability is not of importance, it is important to be able to calculate the averages. For example the average of the square of the Cartesian component $p_{x}$, say with the phase space density $\rho_{2,0}$ is given by 


$$
<p_{x}^{2}>=\int d \Omega d p_{\theta} d p_{\phi}\left[p_{\phi} \sin \phi+p_{\theta} \cos \theta \cos \phi\right]^{2} \rho_{2,0}\left(\theta, \phi, p_{\theta}, p_{\phi}\right)=\frac{22 \pi}{7}
$$

where $p_{r}=0$ was set. 


\section{REFERENCES}

[1] See any standard textbook on angular momentum, e.g. R. N. Zare, Angular momentum, Wiley, (1988)

[2] H. Skenderovic and S. D. Bosanac, Zeit. f. Phys. D35, 107, (1995)

[3] E. P. Wigner, Phys. Rev. 40, 749, (1932)

[4] H.-W. Lee, Phys. Repts. 259, 147, (1995)

[5] J. E. Moyal, Proc. Camb. Phil. Soc. 45, 99, (1949)

[6] M. Gadella, Fortschr. Phys. 43, 229, (1995)

[7] J. G. Muga, R. Sala and R. F. Snider, Phys. Scr. 47, 732, (1993)

[8] R. Sala, S. Brouard and J. G. Muga, J. Chem. Phys. 99, 2708, (1993)

[9] N. L. Balazs and A. Voros, Ann. Phys. 199, 123, (1990)

[10] E. J. Heller, J. Chem. Phys. 65, 1289, (1976)

[11] H. W. Lee and M. O. Scully, J. Chem. Phys. 77, 4604, (1982)

[12] L. Bonci, R. Roncaglia, B. J. West and P. Grigolini, Phys. Rev. A45, 8490, (1992)

[13] M. V. Berry, Phil. Trans. Roy. Soc. 287, 237, (1977)

[14] A. Bonasera, V. N. Kondratyev, A. Smerzi and E. A. Remler, Phys. Rev. Lett. 71, 505, (1993)

[15] A. Royer, Phys. Rev. A43, 44, (1991)

[16] N. Došlić and S. D. Bosanac, Zeit. f. Phys. D32, 261 (1995)

[17] S. D. Bosanac, Phys. Rev. A50, 2899 (1994)

[18] N. Došlić and S. D. Bosanac, Phys. Rev. A51, 3485 (1995)

[19] M. Hillery, R. F. O`Connell, M. O. Scully and E. P. Wigner, Phys. Rep. 106, 122 (1984)

[20] P. Carruthers and F. Zachariasen, Rev. Mod. Phys. 55, 245 (1983)

[21] N. Došlić and S. D. Bosanac, Mol. Phys. A90, 599 (1997)

[22] S. D. Bosanac, Classical Dynamics with the Uncertainty Principle, in From Simplicity to Complexity: Information, Interaction, Emergence, Edited by: A. Mueller, K. Mainzer and W. Saltzer, Vieweg-Verlag, Wiesbaden (1997)

[23] N. Klipa and S. D. Bosanac, Int. J. Theor. Phys., Group Theory and Nonlinear Optics 7, 15, (2000) (http://arXiv.org/abs/quant-ph/0010089)

[24] S. D. Bosanac, Physica Scripta 57, 171, (1998)

[25] T. W. Koerner, Fourier Analysis, Cambridge University Press (1988) 


\section{FIGURES}

FIG. 1. Phase space density for "soft" rigid rotor, for the angular momentum indices $\mathrm{l}=\mathrm{m}=0$ 


\section{TABLES}

\begin{tabular}{|c|c|c|c|}
\hline \hline \hline$l, \mu$ & $\rho_{0, l, \mu} \cdot \pi^{3} e^{E}$ & $<\vec{L}>$ & $<L^{2}>$ \\
\hline 0,0 & 1 & 0 & $0+\frac{3}{2}$ \\
\hline 1,0 & $-1+2 E_{z}$ & 0 & $1 * 2+\frac{3}{2}$ \\
\hline 1,1 & $-1+E-E_{z}+2 L_{z}$ & $1 \hat{z}$ & $1 * 2+\frac{3}{2}$ \\
\hline 2,0 & $1-\frac{2}{3}\left(E+3 E_{z}\right)+\frac{1}{3}\left(E-3 E_{z}\right)^{2}+\frac{8}{3} L^{2}-4 L_{z}^{2}$ & 0 & $2 * 3+\frac{3}{2}$ \\
\hline 2,1 & $\left(1-2 E_{z}\right)\left(1-E+E_{z}-2 L_{z}\right)$ & $1 \hat{z}$ & $2 * 3+\frac{3}{2}$ \\
\hline 2,2 & $-1+\frac{1}{2}\left[E-E_{z}-2\left(1-L_{z}\right)\right]^{2}$ & $2 \hat{z}$ & $2 * 3+\frac{3}{2}$ \\
\hline \hline
\end{tabular}

TABLE I. Phase space density for the ground vibrational state of harmonic oscillator for the first few values of angular momentum numbers. Appropriate angular momentum and its modulus squared are given. Definition of the variables is given in the text.

\begin{tabular}{|c|c|c|c|}
\hline \hline \hline,,$\mu$ & $\rho_{0, l, \mu} \cdot \pi^{3} e^{E}$ & $<\vec{L}>$ & $<L^{2}>$ \\
\hline 0,0 & $1-\frac{1}{3}\left(4 E-2 E^{2}+8 L^{2}\right)$ & 0 & $0+\frac{3}{2}$ \\
\hline 1,0 & $-1+\frac{2}{5}\left(\begin{array}{c}2 E-E^{2}+9 E_{z}-8 E E_{z}+ \\
2 E^{2} E_{z}+12 L^{2}-8 E_{z} L^{2}-8 L_{z}^{2}\end{array}\right)$ & 0 & $1 * 2+\frac{3}{2}$ \\
\hline 1,1 & $-1+\frac{1}{5}\left(\begin{array}{c}13 E-10 E^{2}+2 E^{3}-9 E_{z}+8 E E_{z}- \\
2 E_{z}^{2}+16 L^{2}-8 E L^{2}+8 E_{z} L^{2}+10 L_{z}- \\
8 E L_{z}+4 E^{2} L_{z}-16 L^{2} L_{z}+8 L_{z}^{2}\end{array}\right)$ & $1 \hat{z}$ & $1 * 2+\frac{3}{2}$ \\
\hline \hline
\end{tabular}

TABLE II. Phase space density for the first excited vibrational state of harmonic oscillator for the first few values of angular momentum numbers. Appropriate angular momentum and its modulus squared are given. Definition of the variables is given in the text. 


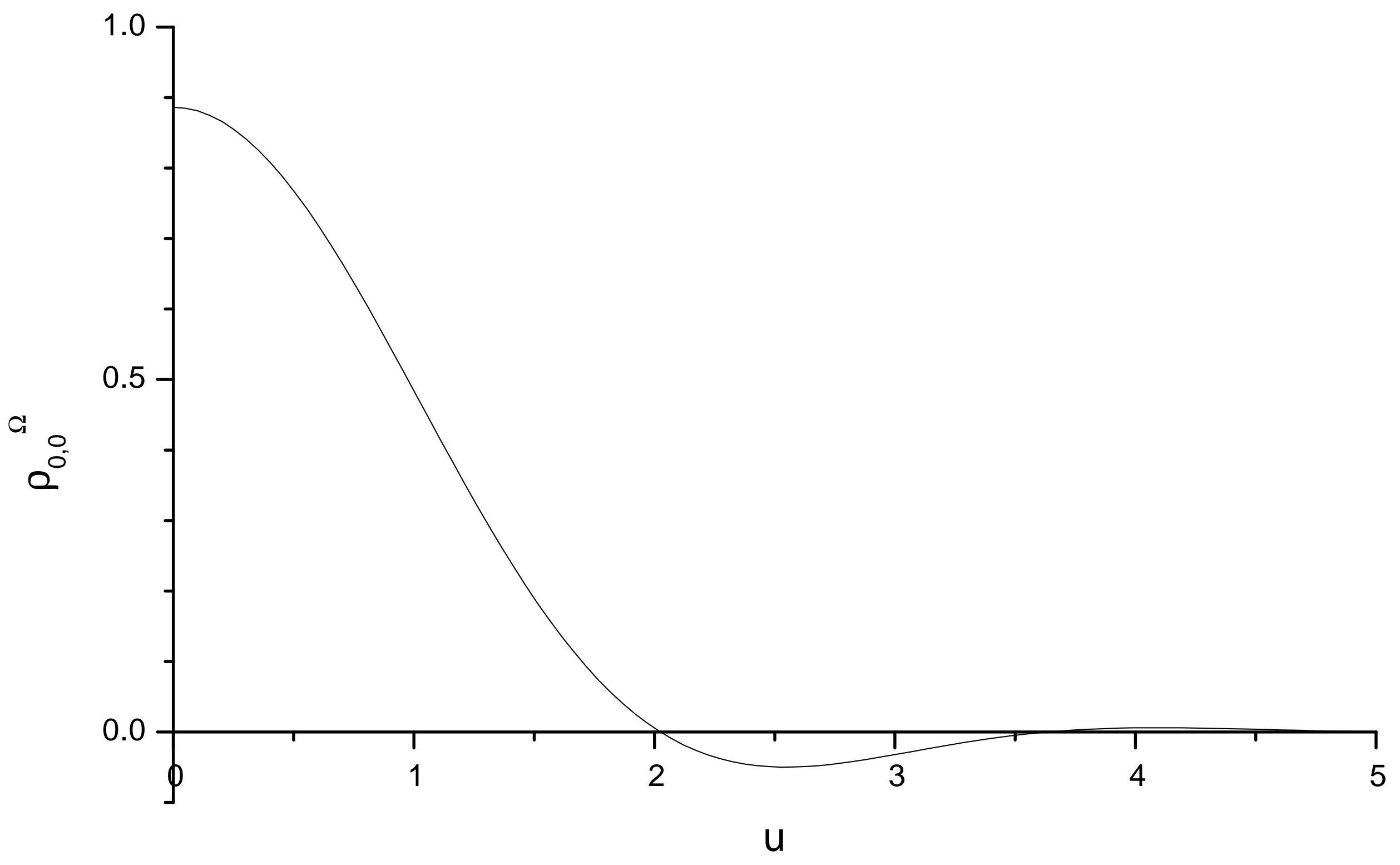

